\title{
Foreword
}

\section{Left Atrial Appendage Occlusion}

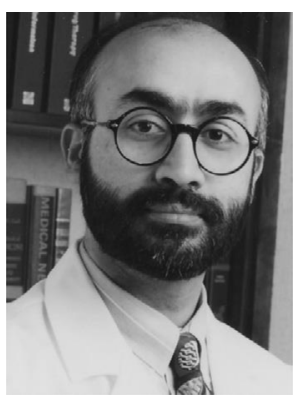

Ranjan K. Thakur, MD, MPH, MBA, FHRS

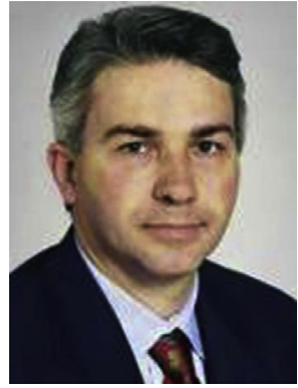

Andrea Natale, MD, FACC, FHRS

Consulting Editors

We are pleased to introduce this issue of Cardiac Electrophysiology Clinics focused on discussion of left atrial appendage occlusion for prevention of embolism in atrial fibrillation (AF).

The readership is well acquainted with the fact that cerebral embolism and other embolic events are some of the most dreaded complications of AF. Anticoagulation can significantly reduce this risk. However, some patients don't tolerate anticoagulation or are poor candidates for it. Stroke prevention can still be achieved in these patients by occluding or removing the left atrial appendage.

The appendage can be occluded surgically (at the time of a concomitant cardiac surgery) or via a number of devices. This issue of Cardiac Electrophysiology Clinics focuses on device-based prevention of embolism. Our knowledge continues to evolve as we learn more about the pathophysiology of $\mathrm{AF}$, the physiologic/anatomic basis of thrombus formation, the association of $\mathrm{AF}$ with systemic vascular disease, the patient and appendage anatomic characteristics in whom the risk-benefit ratio is optimal for device-occlusion therapy, the role of the left atrial appendage in the pathogenesis of $A F$ as well as its contribution to hemodynamics. An increasing number of devices have become available, and we have gained follow-up data, so we now have information on device-related complications to help guide device selection and risk-benefit analysis.

This issue of Cardiac Electrophysiology Clinics has been edited by two interventional cardiologists and an electrophysiologist. We congratulate Drs Holmes, Kar, and Lakkireddy for bringing a balanced perspective to the readership. We hope the readership will find this issue useful and informative.

Ranjan K. Thakur, MD, MPH, MBA, FHRS Sparrow Thoracic and Cardiovascular Institute Michigan State University

1440 East Michigan Avenue, Suite 400 Lansing, MI 48912, USA

Andrea Natale, MD, FACC, FHRS Texas Cardiac Arrhythmia Institute

Center for Atrial Fibrillation at

St. David's Medical Center 1015 East 32nd Street, Suite 516

Austin, TX 78705, USA

E-mail addresses:

thakur@msu.edu (R.K. Thakur) andrea.natale@stdavids.com (A. Natale) 